DOI: $10.20535 / 2410-8286.164697$

\title{
THE IMPACT OF TRANSDISCIPLINARITY ON SIGHT TRANSLATION TRAINING IN SCIENTIFIC AND TECHNICAL DOMAINS
}

\author{
Natalia Saienko \\ National Technical University of Ukraine "Igor Sikorsky Kyiv Polytechnic Institute”, Ukraine \\ saenko106@gmail.com \\ Iryna Simkova \\ National Technical University of Ukraine "Igor Sikorsky Kyiv Polytechnic Institute”, Ukraine \\ isimkova@ukr.net
}

\begin{abstract}
The paper aims to explore the impact of interdisciplinarity on sight translation training in scientific and technical domains. The authors concentrate on the distinctive features of transdisciplinarity and explain the concept of "weak" (used as a practical means) and "strong" transdisciplinarity (complex impact through several levels of reality). 80 future translators/interpreters took part in the research. The results of educational process observations during sight translation classes are presented. Three levels of competences in translating and interpreting (high, medium and low) are identified to conduct the quantitative and qualitative analysis of experimental data during sight translation training. The theoretical and practical analyses, generalisation and mathematical statistics methods were used to stipulate the impact of transdisciplinarity on sight translation training. This research has clearly shown that application of a transdisciplinary approach gives future translators/interpreters opportunity to receive cross-sectorial training in specific fields. Transdisciplinary in sight translation trainings has a positive impact on the development of students' professional identity.
\end{abstract}

Keywords: future translators/interpreters; sight translation training; scientific and technical domains; transdisciplinary approach; transdisciplinarity; competence.

\section{Introduction}

The level of international communication has risen due to the rush development of transport, information and communication, cultural and educational life of society. Nowadays Excellent Science is one of the priority directions in international cooperation. Its aims are to support constructive ideas, to provide researchers from developing countries with the access to up-to-date research infrastructure, and to finance research collaboration in order to implement promising scientific and technological innovations.

Modern scientists are ready for mutual cooperation as far as it helps in searching the ways and means of solving global problems. The fact that global issues in scientific and technical domains can be effectively resolved with international efforts is a powerful stimulus for the development of sight translation training in scientific and technical domains. Taking into account the facts mentioned above, sight translation plays the important role not only in the scientific but political and cultural life of every country.

The information about cutting-age technologies in the scientific and technical fields is spreading through the translation from one (source) language into the other (target) language. Therefore in some cases, the process of sight translation conducting in scientific and technical domains became a way of categorisation and vision of reality. As a result in recent years, scientists have begun to understand clearly that the main difficulties in translation and interpreting are not only the differences in the rules of grammar, functional language and terms in specific technical field, but the differences in the transferring and perception of scientific and technical information in different countries. It means that requirements and accuracy of translation have been growing. When making sight translation in scientific and technical domains, professionals cannot be satisfied with the adequacy of the sight translation "in general", they are to be sure about the uniform impact of the target text on all participants of the process. How to reach such an effect? The answer can be found in the origins of a transdisciplinary approach.

Using a transdisciplinary approach provides the possibility to combine the requirements of the exact sciences, on the one hand, and the introduction of the discourse of psychological, pedagogical, linguistic and translation sciences on the other hand. In the philosophy of science, the construct of "transdisciplinarity" is considered as soft and flexible tools of knowledge.

Thus, we assume that using transdisciplinarity as a sine qua non in sight translation training of future translators/interpreters in scientific and technical domains enables them to acquire the subject knowledge and the knowledge of terminology in scientific and technical fields. As an example, we consider two fields: the computer technologies and nanotechnologies. They were chosen with respect to their nature and specific features.

Nanotechnology and computer technologies are considered as an interdisciplinary field of science and technology. First deals with the study of characteristics of objects and the development of devices with basic 
structural elements of several tens of nanometers. Nevertheless the prefix nano- does not mean only a smaller size of materials, in most cases, it is connected with chemical and physical properties of substances that are invariant with respect to quantity and size. The second field is in a stage of rapid development and has gained widespread recognition as one of the most promising area of scientific and technological development.

The most famous up to date developments in the field of computer technology are androids and humanoid robots, smart devices, 3D printing, electric cars such as Tesla, Lotus, GinzVelo etc. Both fields are the objects of priority government support in many countries all around the world.

\section{Theoretical background}

In Oxford Interdisciplinary Textbook (Frodeman, 2017) we can see that transdisciplinarity is associated with the integrity of knowledge and the extension of the knowledge integration paradigm framework. The theoretical backgrounds of the transdisciplinary approach stemmed from the emergence of science beyond the limits of academic disciplines. Transdisciplinarity is a move through disciplines.

According to the transdisciplinary approach, the production, scientific technology and the development of knowledge are carried out in the new format. In the pedagogical system, where sight translation training of future translators/interpreters takes place, it is necessary to pay attention to future professional activities forecasts. Within the framework of the transdisciplinary approach, there is no predetermined matrix of experimental thinking and methods. The methodology is built up within the process of the study. Thus, the process of pedagogical system design is based on the identification of possible implications of the obtained knowledge and consideration of the promising trends for further developments in scientific and technical fields.

The epistemology of the complexity proposed by Morin (2014) can be considered as the most striking example of a transdisciplinary approach. In our study, the pedagogical system for the sight translation training in scientific and technical fields presupposes that future translators/interpreters exchange the concepts and ideas from different fields of knowledge and in this case, each branch continues to maintain its sovereignty. The important role of transdisciplinarity in sight translation training is its ability to create new metacognitive, cognitive and communicative strategies, which flow from one field to another, ignoring the disciplinary boundaries. It means that each branch loses its cognitive hardness, and problem solving is provided by joint efforts of the branches from various fields.

A lot of researchers treat transdisciplinarity from different points of view. As a result, there are several distinctive features of transdisciplinarity:

1) it emphasises the integration of knowledge, its centeredness (Mokiy, 2017);

2) it provides holism and mobility of knowledge (Klein, 2015);

3 ) it shows that the completeness and integrity of knowledge is not necessarily limited by its centrist organisation (Mokiy, 2017);

4) it is a way of expanding the scientific world outlook, where one or another phenomenon are not limited within one scientific discipline (Mokiy, 2017).

In this paper, we consider transdisciplinarity as a research strategy that crosses disciplinary boundaries and develops a holistic vision of a problem.

Jeder (2014) investigates the phenomenon of transdisciplinarity and shows a rather eclectic mix of projects and new structures created primarily to address the challenges associated with the transdisciplinary nature in vocational training. Specific examples of the convergence of the required knowledge from various fields that we can observe when implementing projects such as CIRET (France), ATLAS (EU), RST (Russia), Td-network (Switzerland), SciTS (Science of Team Science) (USA) indicate the relevance of the application of the transdisciplinary approach when it comes to preparing future translators/interpreters for the sight translation performance in the scientific and technical domains.

Nicolescu (2012) suggests that the structure of the real world has a form of stratification. Each layer is an integer of the existing one. There are four main layers: physical, material, organic, and spiritual. Each of these layers has its own laws and principles. A higher layer of existence is entirely based on the low, but it is determined only partially. Therefore designing metaphysics on one principle or one set of principles is impossible. The constructed pictures of the world unity are not right. Neither "metaphysics from below" nor "metaphysics from above" is enough as far as there is a natural system of the world that is not constructed. Its structure is viewed as a phenomenon that cannot be reduced to centralised unity, or to the root cause or the ultimate goal.

Usually, categorical laws include four groups of basic laws: meaning, coherence, stratification, and dependence (Mokiy, 2017). However, many scholars (Nicolescu, 2012; Popa, 2015) focus their attention on the third group - the laws of stratification. 
According to the transdisciplinary approach levels of cognition (Nicolescu, 2012) are independent of each other. Although there is a hierarchy of categories, laws, levels, subconsciousness of social levels, the fundamental principles at one level may be considered as uncertain at other level. Each level has its own "local autonomy". These properties determine the problem of application, for example, of higher levels of cognition to phenomena that are observed at lower levels.

In this case, Max-Neef (2005) proposes the concept of "weak" and "strong" transdisciplinarity. Weak transdisciplinarity is used as a practical means to address systematically to different problems. When using weak transdisciplinarity in not enough, strong transdisciplinarity is used both as a tool and as a project. As a rule disciplinary researches address only one level of reality. Transdisciplinarity can provide a more complex impact through several levels of reality, and in the case of strong transdisciplinary effects, the effect is realised through several structural levels (Max-Neef, 2005).

Darbellay (2015) considers transdisciplinarity as a form of knowledge integration, which involves close internal communication with the researcher's professional experience and subconsciousness. Mokiy (2017) distinguishes in the transdisciplinary approach the basic preconditions: metaphorical interconnections, formal relationships between applied sciences, closer internal connections between researcher's professional experience and his/her subconscious, metaphors that gains fundamental cognitive significance, and transdisciplinary (universal) worldview.

The transdisciplinarity in modern science reflects a new synthesis of scientific problems in relation to their canonical classification, which has objectively-structural foundations or historical and scientific grounds. The transdisciplinary studies of certain types in their pure form are the prior stage of the application of the transdisciplinary approach. They prepare the basis for the higher stage of its (approach) application associated with the formation and further development of the corresponding scientific theory or even the scientific branch.

The research and the formation of scientific theories and scientific fields of two or more classical sciences is a substantially more basic form of a transdisciplinary approach implementation than conducting complex studies or the formation of complex theories and sciences. The first form of the transdisciplinary approach is aimed at detailing the classical classification of sciences. It is associated with gaps' filling in it at the intersection of disciplines, just as the introduction of an irrational number is considered as a "gaps' filling" in the set of rational numbers.

At the same time, the transdisciplinary approach (in its complex form) goes beyond the canonical classification of sciences. It is aimed at the formation of a substantially new synthesis of scientific problems, which gives rise to a new classification of complex sciences. However, the interaction between different forms of transdisciplinary approach should be mentioned. It generates a synergistic connection during the process of modern science development and gives grounds to speak about the application of the transdisciplinary approach in general.

\section{The features of scientific and technical texts}

A distinctive feature of scientific and technical texts, both in English and in Ukrainian, is their practical purpose. One of their main functions is to meet the information needs or provide instructions to the recipient so that he can handle the technical tasks, since instructions, technical developments, and scientific articles are not read in order to obtain aesthetic pleasure (Byrne, 2014). Scientific and technical texts are usually written in a specialised language and using terms, functional language etc. Nevertheless, it is not their main characteristic. The main characteristic of scientific and technical texts is their practical purpose, with the help of which users receive information that enables them to complete tasks or solve problems related to the applied sciences.

The lack of implicit knowledge, lack of knowledge about how to transfer the relationship between terms in the source and target language, lack of knowledge about how to transfer quantitative indicators, lack of knowledge in terminology, lack of knowledge about the "weaknesses" of the source text (Klein, 2015) are considered as the main difficulties associated with sight translation performance in the scientific and technical domains.

So, the aim of the paper is to study the theoretical background of the transdisciplinary approach and explore its impact on sight translation training in scientific and technical domains.

\section{Methods}

General Background of Research

To achieve the goals of the study the empirical and theoretical methods were used. The theoretical methods used in the study are the generalisation of philosophical, psychological, pedagogical and linguistics 
literature to consider the theoretical backgrounds of transdisciplinary approach; the comparative analysis to stipulate the distinctive features of transdisciplinarity; the description of the results to explain the role of transdisciplinarity in scientific and technical fields.

The empirical methods used in the study are the observation of the educational process in those classes where transdisciplinary approach took place; the quantitative and qualitative analysis of experimental data to show the impact of transdisciplinarity on the sight translation training in scientific and technical domains, non-parametric criterion of Shapiro-Wilcoxon for checking the significance of impact.

\section{Participants}

To illustrate the applicability of considered above approach for sight translation training, the study was carried out among 80 future translators/interpreters. The future translators/interpreters are students of the fourth year of studying from the Faculty of Linguistics Igor Sikorsky Kyiv Polytechnic Institute. The sample was divided into four groups of students (LA-41, LA-42, LA-43, and LA-44) that were of similar size (20 students). Four groups participated in the testing studying from September 2017 to May 2018.

Two testing groups of future translators/interpreters participated in sight translation training where the transdisciplinary approach was applied and two academic groups of future translators/interpreters experienced ordinary sight translation training (when only linguistic or translation approaches were used).

\section{Instrument and Procedures}

As was mentioned to verify the significance of impact of transdisciplinarity on the sight translation training in scientific and technical domains the method of Shapiro-Wilcoxon (non-parametric criterion) was used.

The empirical data that forming Intervals below were given due to "learning quotient" formula:

$$
L Q=\frac{\mathrm{Ip}}{\mathrm{Tp}}
$$

where LQ means learning quotient, Ip means the total number of points got by a student, Tp means the maximum number of points that could be get by a student. This formula was used to identify the "learning quotient" of future translators/interpreters from testing and academic groups.

Taking into account the fact that some of the values occur only once in one sample (for instance, the value 0.48 ) while other values (for instance, value 0,2 ) occur in four or three samples at the same time, we came to the conclusion that it is impossible to use each individual sample value for Shapiro-Wilcoxon method. Therefore, we adapted our calculation according to the Shapiro-Wilcoxon method requirements. Instead of analysing each individual sample value frequency; we decided to form Intervals, which cover all values occurred in the samples.

So, it is suggested to use four Intervals equal in length according to the "learning quotient" results in testing groups:

Interval $1=[0,6 ; 0,72)$

Interval $2=[0,72 ; 0,8)$

Interval $3=[0,8 ; 0,92)$

Interval $4=[0,92 ; 1)$.

And four Intervals equal in length according to the "learning quotient" results in academic groups:

Interval $1=[0,08 ; 0,2)$

Interval $2=[0,2 ; 0,28)$

Interval $3=[0,28 ; 0,4)$

Interval $4=[0,4 ; 0,6)$.

Wilcoxon method helps us to compare values occurs in samples. This method is used in groups where the number of students is less than 25. After receiving the experimental data the absolute values of the shifts are ranked and then the ranks are summed.

To identify minimum and maximum values the following formulas are used:

$\mathrm{W}=\mathrm{n}(\mathrm{n}+1) / 2$ where $\mathrm{n}$ is the range of the second sample to identify minimum value.

$\mathrm{W}=\mathrm{n}(\mathrm{n}+1) / 2+\mathrm{mn}$ where $\mathrm{n}$ is the range of the second sample, $\mathrm{m}$ is the range of the first sample. It is used to identify the maximum value.

If shifts in one direction or another occur randomly, then the sum of their ranks will be approximately equal. The impact is significant if the intensity of the shifts in one direction is higher and the sum of the ranks of the absolute values of the shifts in the opposite direction is lower than it could be with random changes (McElreath, 2015).

According to Shapiro-Wilcoxon method, the impact is significant if values in the Intervals in each group are equal or higher than 0,6 . If values in samples are equal or higher than 0,6 , it means that future 
translators/interpreters have made significant progress or show steady improvement in skills. If values in samples are lower than 0,6 , it means that future translators/ interpreters have some difficulty achieving good results in some activities.

\section{Results}

The quantitative and qualitative analysis of experimental data indicates that testing groups where the transdisciplinary approach was applied during sight translation training demonstrate better results than academic groups with ordinary methods in sight translation training.

Table 1 shows that all Intervals in testing groups are equal or higher than 0,6 , whereas values in academic groups are lower than 0,6 . It proves that the impact of transdisciplinarity on the sight translation training in scientific and technical domains is substantial.

Table 1

\section{Data about value and intervals in testing and academic groups}

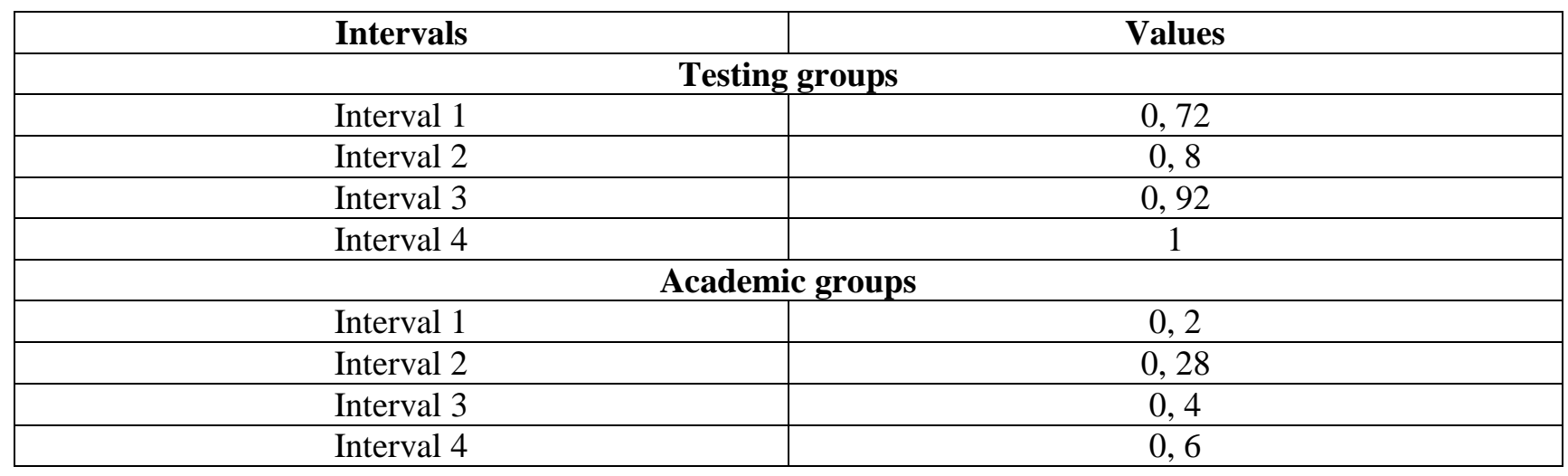

Fifure 1 shows that 15 students in testing group 1 (TG-1) and 16 in testing groups 2 (TG-2) possess a high level of translators' competence. It is bigger than in academic group 1 (AG-1), where only 10 students possess a high level of translators' competence and in academic group 2 (AG -2) where 8 students possess a high level of translators' competence.

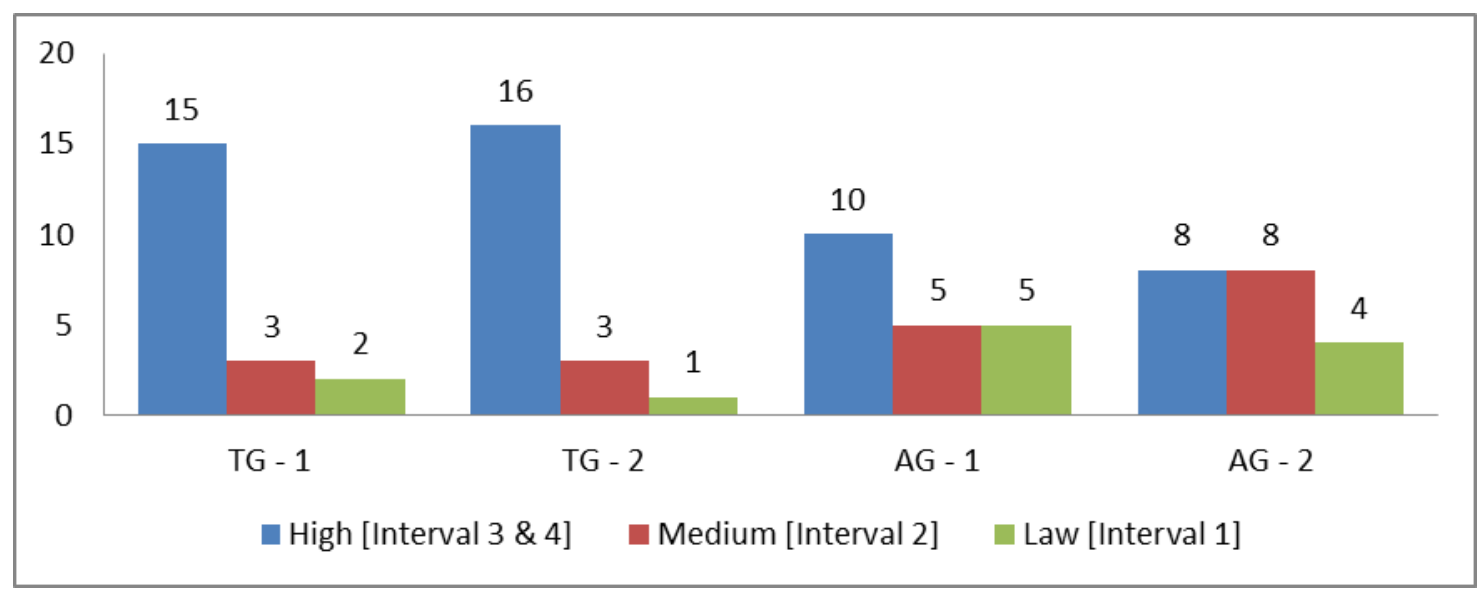

\section{Figure 1. The impact of transdisciplinarity on the sight translation training in scientific and technical domains}

The correlation between experimental data in Fig. 1 and intervals in testing and academic groups in Table 1 can be traced as follows. As exemplified in testing group 1 the results that are demonstrated by 2 students with low level are located in the range of Interval 1, the results that are demonstrated by 3 students with medium level are located in the range of Interval 2, and the results that are demonstrated by 15 students with high level are located in the range of Intervals 3 and 4.

An important feature of the current stage of scientific and technological progress lies in transdisciplinarity and as a consequence of the mutual penetration of special terminology from one field of knowledge to others (Simkova, 2018b). Our observations of the educational process during sight translation classes shows that in order to translate/interpret technical literature and documentation, for example, from computer technology field, it is necessary for future translators/interpreters simultaneously use different 
dictionaries in technology, radio electronics, microelectronics, computing, economics and finance, and even advertising and marketing.

During the observation process there was a possibility to interview the future translators/interpreters. The frequently asked questions were: "Do you know the procedure of sight translations in scientific and technical domains?", "What material do you usually use to perform sight translation in scientific and technical domains?" etc. The interview with future translators/interpreters shows that various set of dictionaries is required during the translation/interpreting of modern scientific and technical journals such as "The Journal of Nanoscience and Nanotechnology", "World of Nanotechnology" etc. Only several students from each group knew about sight translation procedure.

Scholars apply different evaluation criteria and different approaches to assess the level of translator's/interpreter's competence. It may be language proficiency, the quality of translation/interpreting as a product (for texts of different functional styles) or as a process (Breddik, 2014). Most of them believe that the main criterion to assess the level of translator's/interpreter's competence is its correlation with the target results.

PACTE experts (Albir, 2017) suggest that translator's/interpreter's competence consists of various subcompetences. Within the scope of sight translation training study, we highlight five sub-competences: bilingual, translation, extralinguistic, personal, and strategic. Thus, the general level of translator's/interpreter's competence can be measured owing to the level of sub-competences formation and results demonstrated by future translators/interpreters during sight translation performance in the scientific and technical domains.

For instance, if translator's/interpreter's competence during sight translation performance in scientific and technical domains of each student takes as 25 points, then each sub-competence takes as 5 points.

To provide the quantitative and qualitative analysis of experimental data during sight translation training in scientific and technical domains we identified three levels of competence in translating and interpreting: high, medium and low (Simkova, 2018a).

The high level (25-20) suggests that the future translator/interpreter shows a complete understanding of the source text, possess the knowledge of translation ethics, teamwork skills, ability to work in stress situations, he/she is able effectively to plan translation and evaluate target texts, helshe is able to transfer the total content of the source text to the target text without long unmotivated pauses.

The medium level (20-15) suggests that the future translator/interpreter demonstrates the correct but incomplete understanding of the source text, lack of translation ethics knowledge, lack of teamwork skills and average ability to work in stressful situations, he/she is able to plan translation and evaluate target texts, he/she is able to transfer about $75 \%$ of source text content into the target text with a small amount of unmotivated pauses.

The low level (15-10) suggests that the future translator/interpreter shows insufficiently correct and accurate understanding of the source text, poor knowledge of translation ethics, low teamwork skills and he/she is unable to work in stress situations, helshe shows inefficient translation planning and low target language evaluation skills, he/she is able to transfer less than $60 \%$ of source text content into the target text with a large number of unmotivated pauses.

If the total points gained by student are below 10, it means that the future translator/interpreter shows incorrect and inaccurate understanding of the source language, ignorance of translation ethical norms, he/she does not know how to work in a team and behave in stressful situations, he/she cannot plan translation and evaluate translation.

\section{Discussion}

It was the main purpose of the paper to draw the attention of the transdisciplinary approach and to explore its impact on sight translation training in scientific and technical domains. We agree with Jeder (2014), who believes that transdisciplinarity is a profitable way to meet the challenges of our time. The main problem in joint research is that translation, linguistic, psychological and pedagogical studies are connected with scientific and technical issues. It creates the combination of various disciplinary knowledge, methods and methodologies. The discussion of specific problems and the coordination of research protocols, the justification of the pedagogical system necessary for the professional training of future translators/interpreters requires the implementation of a transdisciplinary approach as far as it provides the possibility to cross boundaries of separate discipline and widen national educational formats (Karnedi, 2015).

The results of comparative analysis of transdisciplinary approach and other approaches to sight translation training show the transformation of social consciousness (Popa, 2015), changing role of the specialist and functions of science due to the transformations in scientific and technical fields. It means that 
teachers and future translators and interpreters come across "open innovation", the formation of a "sociality of a new type" and bioethical ideology.

Our results are in good agreement with Max-Neef (2005), Mokiy (2017), Darbellay (2015). In this paper, we provide principal provisions of the transdisciplinary approach aimed at the identification of interaction between the psycho-pedagogical system, the scientific and technical sphere and the translation and linguistic aspects. For this purpose we consider three interrelated issues: 1) to define the effective pedagogical conditions for the sight translation training, 2) to identify the priority innovative branches (computer technologies and nanotechnologies) in scientific and technical domains that can be used for sight translation training; 3) the involving of future translators/interpreters in the sight translation training design and implementation.

That is why we believe that transdisciplinarity is seen as a resource for the synergy of the perspectives of academic (pedagogical, translational and linguistic) and applied (psychological and scientific and technical) types of knowledge in solving problems. In our study the transdisciplinary approach helps to solve the epistemological problem. It is able to help future translators/interpreter not only to possess better knowledge of the source and target languages in scientific and technical domains, but also heighten mutual understanding of different cultures and different personalities, different thinking patterns and different levels of technical development.

Using of transdisciplinary during sight translation training solves three questions: the formation of an object of research in various perspectives; activation of communication between representatives of different sciences; introduction of the results of interdisciplinary research into practice, as well as examination of scientific results in the disciplinary knowledge system.

Usually, these phenomena are described by sociologists and philosophers with the help of theoretical models including "post-academic models" (Millar, 2013), "post-neoclassical models" (Stolyarova, 2015), "models of the society knowledge" (Frodeman, 2017), etc. We believe that the application of the transdisciplinary approach hardens connections between the scientific and technical domains and the translators' experience. These connections were weakened as far as the difference between the scientific and technical domains and the linguistic and pedagogics is obvious (Mokiy, 2017).

The application of the transdisciplinary approach in the process of sight translation training generates new phenomena, such as Triple Helix: Sight translation - Scientific and technical domains - Pedagogical conditions. The emergence of the Triple Helix is associated with the substantial changes in science, technology, economics and politics that in its turn are influenced by globalisation. It is considered as efficient way of interaction between three subjects: Translators - Educators - Scientists.

One of the real life cases is when IT specialists are asked to prepare presentations on one of the special topics (Data mining, LAN Networks, Programming languages etc.) for non-specialists. Then before starting professional training on special topic teachers provide future translators/interpreters with these presentations. It helps future translators/interpreters stay informed about up-to-date IT trends and enhances the possession of IT terms. The innovation system evolution takes place at the intersection of two different areas (subjects, representatives etc.) that are not identical to each other, for example, translators/interpreters and scientists. The separate activity of each subject does not bring the effective result. So, if the activity of Double Helix is not effective, the emergence of the Triple Helix is inevitable.

Thus, the implementation of Triple Helix encourages the creating of new knowledge. They occupy a dominant position in the system of innovative development as far as modern science development produces synthetic areas, which include both classical (canonic) and applied interdisciplinary researches. In this case, transdisciplinarity initiates the formation of "clusters" that form the future potential of innovation development (for instance, nanotechnologies, information technologies etc.) and create connections between scientists, translators and educators (Vaivode, 2015).

Modern requirements to the quality of higher education are aimed at high achievements in the innovative areas of science and technology. It is known that scientific and technological achievements are usually carried out at the intersection of disciplines (for example, nanotechnology, chosen by us as one of the branches) as a result of complex research of objects and related problems. Typically, transdisciplinary research involves various scientific disciplines in order to comprehensively study a single subject, for instance, the formation of future translators/interpreters competence in sight translation in scientific and technical domains.

As much as we consider a question of sight translation training in the scientific and technical domains, the cognitive activity of future translators/interpreters goes beyond the scope of translation and is combined with modern advances in science and technology. Therefore the application of the transdisciplinary approach is logical and essential. A transdisciplinary approach is not only a joint study of problems in various fields of 
science. The first element of the characteristics of the transdisciplinary approach is the joint study of the problems of various, remote branches of science. Sometimes it is characterised by quite different forms and means of research and put forward different requirements for scientific research.

The application of the transdisciplinary approach enhances the level of professional, ideological, communicative and intercultural competency of specialists. At the present stage of the development of education and science, the problem of general scientific methodology becomes very urgent, where, along with the processes of differentiation of scientific knowledge in the branches, its integration becomes important (Nicolescu, 2012; Millar, 2013).

Some authors (Popa, 2015; Darbellay, 2015) suggest a typology of transdisciplinary approaches depending on the field of research. It is based on the differences between descriptive-analytical and transformational approaches (Popa, 2015). So, when applying the transdisciplinarity in sight translation training of future translators/interpreters in the scientific and technical domains, we are referring to the basics that are not represented by one branch of knowledge. We are addressing a socially relevant problem, for which there are several approaches, which require the coordination of strategies and methods for its consideration for further resolution.

Therefore, the transdisciplinary approach is associated with the implementation of different (sometimes opposite) trends. It is aimed at scientific differentiation; it means the intersection of complex sciences that combine problems from various canonical sciences. Applying the transdisciplinary approach in sight translation training of future translators/interpreters in the scientific and technical domains creates new boundaries in the plane of scientific problems of professional training of translators/interpreters.

Taking into account all the facts discussed above, we can conclude that the application of the transdisciplinary approach in sight translation training of future translators/interpreters in scientific and technical domains requires the creation of new types of pedagogical conditions, which is a prerequisite for the achievement of higher forms of professionalism. If at the beginning of the sight translation training in the scientific and technical domains the knowledge from various fields possessed by translator/ interpreter can be defined as "chaos", then, with the application of the transdisciplinary approach, their knowledge structuralised, organised, and ordered according to the structural scientific connections (Pauliuk \& Hertwich, 2015).

Consequently, the impact of transdisciplinary on sight translation training of future translators/interpreters is clearly traceable. Students in academic groups develop only the knowledge, skills and abilities necessary for the implementation of sight translation. While the implementation of transdisciplinarity in testing groups helps students acquire substantive knowledge in the field of nanotechnology and computer technology; their obtaining knowledge is not limited to learning terms or their comparative analysis. Thanks to transdisciplinarity future translators/interpreters receive cross-sectorial training in specific fields. We can monitor the impact of transdisciplinary in sight translation trainings as far as it influences their continuing professional development and professional identity development. Students get an opportunity to learn about the tools of professional translators/interpreters development and to design their own model of professional planning.

\section{Conclusion}

Thus, the transdisciplinary in sight translation training of future translators/interpreters in scientific and technical domains is considered as a form of scientific knowledge organisation based on certain links between scientific fields (branches of knowledge), methods and technologies that provide solutions to complex scientific and technical problems. It (transdisciplinary nature) is characterised by the properties of the integrability of disciplines based on the transfer of research methods from one discipline to another. The application of such an approach during the sight translation training of future translators/interpreters in scientific and technical domains requires synthesis of the results obtained through various scientific disciplines.

The transdisciplinary approach in modern science comprises various forms of studies. It integrates studies connected with conducting inter-canonical (borderline) and complex (related to different order and type) studies, as well as the formation of a corresponding type of scientific theories and scientific fields of knowledge. All of them have specific objective grounds, and, thus, have a specific significance for the development of scientific knowledge during sight translation training of future translators/interpreters in scientific and technical domains.

\section{Acknowledgements}

This research was supported by the Department of English Language for Humanities and Faculty of Linguistics, Igor Sikorsky Kyiv Polytechnic Institute, Ukraine. 


\section{References:}

Albir, H. (Ed.). (2017). Researching Translation Competence by PACTE group. [Benjamins TranslationLibrary, 127]. AmsterdamPhiladelphia: John Benjamins. http://dx.doi.org//10.1075/btl.127

Breddik, U. (2014). Management in an Organization. N. Y.: Springer.

Byrne, J. (2014). Scientific and Technical Translation Explained. Rutledge.

Darbellay, F. (2015). Rethinking inter- and transdisciplinarity: Undisciplined knowledge and the emergence of a new thought style. Futures, 65, 163-174. http://dx.doi.org/10.1016/j.futures.2014.10.009

Frodeman, R., Klein, J., \& Pacheco, R. (2017). The Oxford handbook of interdisciplinarity. Oxford: Oxford University Press.

Jeder, D. (2014). Transdisciplinarity - the advantage of a holistic approach to life. Procedia - Social and Behavioral Sciences, 137, 127-131. http://dx.doi.org/10.1016/j.sbspro.2014.05.264

Karnedi, D. (2015). Translating economics textbooks: a case study of epistemicide. TEFLIN Journal, $26(1), 59-84$. http://dx.doi.org/10.15639/teflinjournal.v26i1/59-84

Klein, J. (2015). Reprint of "Discourses of transdisciplinarity: Looking Back to the Future". Futures, 65, 10-16. doi.org/10.1016/j.futures.2014.08.008

Max-Neef, M. (2005). Foundations of transdisciplinarity. Ecological Economics, 53(1), 5-16. http://dx.doi.org/10.1016/j.ecolecon.2005.01.014

McElreath, R. (2016). Statistical Rethinking: A Bayesian Course with Examples in R and Stan. N. Y.: Chapman \& Hall.

Millar, M. (2013). Interdisciplinary research and the early career: The effect of interdisciplinary dissertation research on career placement and publication productivity of doctoral graduates in the sciences. Research Policy, 42 (5), 1152-1164. http://dx.doi.org/doi.org/10.1016/j.respol.2013.02.004

Mokiy, V. (2017). Metodologiya transdisciplinarnosti-4 [Methodology of Transdisciplinarity-4]. Nalchik: Russian school of Transdisciplinarity; Institute of Transdisciplinary Technologies. Retrieved January 26, 2019 from http://www.anoitt.ru/tdbiblioteka/tdmetodol.php

Morin, E. (2014). Le Paradigme perdu: la nature humaine [Paradigm lost. Human nature]. Paris: Éditions du Seuil.

Nicolescu, B. (2012). Transdisciplinarity: the hidden third, between the subject and the object. Human \& Social Studies. Research and Practice, 1 (1), 13-28. http://dx.doi.org/10.2478/v10317-012-0002-5

Pauliuk, S. \& Hertwich, E. (2015). Socioeconomic metabolism as paradigm for studying the biophysical basis of human societies. Ecological Economics, 119, 83-93. http://dx.doi.org/10.1016/j.ecolecon.2015.08.012

Popa, F., Guillermin, M., \& Dedeurwaerdere, T. (2015). Pragmatist approach to transdisciplinarity in sustainability research: From complex systems theory to reflexive science. Futures, 65, 45-56. http://dx.doi.org/10.1016/j.futures.2014.02.002

Simkova, I. (2018a). The criteria for professional translators' competence assessment. International Journal of Innovative Technologies in Social Science, 6(10), 3-7. http://dx.doi.org/10.31435/rsglobal_ijitss/30082018/6103

Simkova, I. (2018b). The effect of transdisciplinary approach on the training of translators and interpreters. World Science. Multidisciplinary Scientific Edition,4 (32), vol.7, 16-20. Retrieved January 26, 2019 from https://ws-conference.com/

Stolyarova, O. (2015). Istoriya i philosophiya nauki versus STS [History and philosophy of science versus STS]. Voprosy hilosophii, 7, 73-78. Retrieved January 26, 2019 from http://vphil.ru/index.php?option=com_content\&task=view\&id=1198\&Itemid=52

Vaivode, I. (2015). Triple Helix Model of University-Industry-Government Cooperation in Context of Uncertainties. Procedia Social and Behavioral Sciences, 213, 1063-1067. http://dx.doi.org/10.1016/j.sbspro.2015.11.526 